\title{
Nonlinear voltage dependence of shot noise
}

\author{
Yadong Wei, Baigeng Wang, and Jian Wang \\ Department of Physics, The University of Hong Kong, Pokfulam Road, Hong Kong, China
}

Hong Guo

Center for the Physics of Materials and Department of Physics, McGill University, Montreal, PQ, Canada H3A 2T8

(Received 19 January 1999)

\begin{abstract}
The electron shot noise in a multiprobe mesoscopic conductor can have a nonlinear dependence on the strength of driving bias voltage. This paper presents a theoretical formulation for the nonlinear noise spectra. We pay special attention to maintain gauge invariance at the nonlinear level. At small but finite voltages, explicit expressions for nonlinear noise spectra, expanded order by order in the bias, have been derived. In the wideband limit, a closed-form solution of the noise spectra for finite voltages is obtained.
\end{abstract}

[S0163-1829(99)01548-9]

\section{INTRODUCTION}

Electric shot noise of mesoscopic systems has been studied extensively ${ }^{1-4}$ because the spectra of it contains information that characterizes transport of the conductor. Shot noise has been used to probe the kinetics of electrons ${ }^{5}$ and to investigate correlations of electronic wave functions. ${ }^{6}$ A classical conductor is characterized by Poissonian noise, ${ }^{7}$ where the current fluctuation $\left\langle(\Delta I)^{2}\right\rangle$ in a frequency range $\Delta \nu$ is proportional to electrical current $I,\left\langle(\Delta I)^{2}\right\rangle=2 q I \Delta \nu$, where $q$ is the electron charge. On the other hand, for a mesoscopic conductor shot noise is influenced by two other factors: the Pauli exclusion principle and the Coulomb interaction. Pauli exclusion reduces Poissonian noise by a factor proportional to $(1-T)$ for each transmission subband, ${ }^{8,9}$ where $T$ is the transmission coefficient of the subband. Coulomb interaction can contribute to reduce or enhance shot noise depending on system details. The Pauli suppression of shot noise has been convincingly demonstrated by several experiments. ${ }^{10-12,4}$ The universal Coulomb suppression of short noise in nondegenerate diffusive conductors has been observed in computer simulations ${ }^{13}$ and confirmed theoretically using BoltzmannLangevin equation. ${ }^{14}$ The quantum enhancement of shot noise from the classical value due to Coulomb interaction has recently been explored experimentally. ${ }^{15,16}$ For a tunneling structure with or without a magnetic field, shot noise versus voltage increases drastically in the region of negative differential resistance (NDR). If one assumes sequential tunneling of the electron transport, ${ }^{17}$ numerical results ${ }^{15}$ were in good agreement with those of experiment, indicating the enhancement of shot noise was indeed caused by Coulomb interaction. The effects of Coulomb interaction has also been studied from the point of view of scattering matrix ${ }^{18}$ where an enhancement of shot noise was found to be related to the multistability when a tunneling system is out of equilibrium.

Shot noise has only been carefully studied in the limit of small external bias where electric current due to each transmission subband is a linear function of bias, $I_{i}$ $=\left(2 e^{2} / h\right) V_{\text {bias }} T_{i}$, where $T_{i}$ is the zero-bias transmission coefficient of the $i$ th subband. However, in a nonlinear device the electric current is a nonlinear function of bias, I
$=I\left(V_{\text {bias }}\right)$. A notable example is the resonance tunneling structure where current $I$ varies with bias in a nonlinear fashion giving rise to NDR. Previous investigations ${ }^{10,16,15,18}$ have already indicated that the nonlinear $I-V$ characteristics have a profound influence on the shot-noise spectra including the enhancement of it.

The purpose of the present work is to report a microscopic theory for calculating shot noise at the nonlinear regime in mesoscopic conductors. Our theory is based on nonequilibrium Green's functions where the electron-electron interaction is treated in a self-consistent manner at the Hartree level. A direct consequence of self-consistency is that shot noise becomes only a function of voltage difference, which is the required physical condition (gauge invariance) for a nonlinear theory. We derive the nonlinear shot-noise formula at zero temperature, which, in the wideband limit, can be exactly evaluated. For more general situations beyond the wideband limit, we derive shot-noise spectra order by order in bias voltage.

In Sec. II we present the derivation of the nonlinear shotnoise spectra. Sections III and IV present the wideband limit result with numerical evaluations, as well as the weakly nonlinear analysis of the shot noise. Section V summarizes the main findings of this work.

\section{NONLINEAR SHOT-NOISE FORMULA}

Several theoretical approachs have been applied to study shot noise including the scattering matrix theory, ${ }^{9}$ semiclassical kinetic theory, ${ }^{19,20}$ and the nonequilibrium Green'sfunction (NEGF) theory. ${ }^{21,22}$ For a full nonlinear analysis of quantum transport in the mesoscopic regime, it is most convenient to use NEGF with the necessary extension by including the internal potential buildup self-consistently. The latter extension is necessary and essential due to electron-electron interactions and due to the gauge-invariance requirement that the noise spectra remains unchanged when voltages at all probes are shifted by the same constant amount. The physics of gauge invariance has been discussed in Ref. 23. Recently, Gramespacher and Büttiker ${ }^{24}$ discussed the relationship between scattering matrix theory and Hamiltonian approach in 
which the transmission coefficient is expressed in terms of Green's function. Since shot-noise spectra $\left\langle\Delta I_{\alpha} \Delta I_{\beta}\right\rangle$ has originally been written in terms of the scattering matrix, ${ }^{9}$ the Hamiltonian approach allows us to rewrite it in terms of the Green's function. We will then supplement it with the necessary steps of determining the internal electrostatic potential buildup using the NEGF.

We consider a quantum coherent multiprobe conductor specified by the Hamiltonian

$H=\sum_{k, \alpha} \epsilon_{k \alpha} c_{k \alpha}^{\dagger} c_{k \alpha}+H_{\mathrm{cen}}\left\{d_{n}, d_{n}^{\dagger}\right\}+\sum_{k, \alpha, n}\left[T_{k \alpha, n} c_{k \alpha}^{\dagger} d_{n}+\right.$ c.c. $]$,

where $\epsilon_{k \alpha}=\epsilon_{k}^{0}+q V_{\alpha}$. The first term of Eq. (1) describes the probes where dc signal is applied far from the conductor; the second term is the general Hamiltonian for the scattering region; the last term gives the coupling between probes and the scattering region with the coupling matrix $T_{k \alpha, n}$. Here $c_{k \alpha}^{\dagger}\left(c_{k \alpha}\right)$ is the creation (annihilation) operator of electrons inside the $\alpha$ probe. Similarly, $d_{n}^{\dagger}\left(d_{n}\right)$ is the operator for the scattering region. It is important to note that we will include the internal Coulomb potential $U$ inside the scattering region so that the actual Hamiltonian of the scattering region is $H_{\text {cen }}+q U$.

The retarded scattering Green's function $G^{r}=G^{r}(E, U)$, where $U=U(\mathbf{r})$ is the electrostatic potential buildup inside the scattering region due to interacting electrons, is given by ${ }^{25}$

$$
G^{r}(E, U)=\frac{1}{E-H-q U-\Sigma^{r}},
$$

where the self-energy $\Sigma^{r} \equiv \Sigma_{\alpha} \Sigma_{\alpha}^{r}\left(E-q V_{\alpha}\right)$ is defined as

$$
\Sigma_{\alpha}^{r}(E)=\frac{1}{2 \pi} \int \frac{\Gamma_{\alpha}\left(E^{\prime}\right) d E^{\prime}}{E-E^{\prime}+i \eta}
$$

and $\eta$ is a positive infinitesimal number and $\Gamma_{\alpha}(E)$ is the linewidth function

$$
\left[\Gamma_{\alpha}(E)\right]_{m n}=2 \pi \sum_{k} T_{k \alpha, m}^{*} T_{k \alpha, n} \delta\left(E-\epsilon_{k \alpha}\right) .
$$

Finally, the scattering matrix can be expressed in terms of Green's functions by Fisher-Lee relation ${ }^{26,24}$

$$
s_{\alpha \beta}=\delta_{\alpha \beta}-2 \pi i W_{\alpha}^{\dagger} G^{r} W_{\beta},
$$

where $W_{\alpha}$ satisfies $2 \pi W_{\alpha} W_{\alpha}^{\dagger}=\Gamma_{\alpha}$.

Substituting Eq. (5) into the shot-noise formula derived in Ref. 9, it is straightforward to derive $\mathrm{e}^{27}$

$$
\begin{aligned}
\left\langle\Delta I_{\alpha} \Delta I_{\beta}\right\rangle= & \frac{q^{2}}{\pi} \Delta \nu \sum_{\gamma, \delta} \int d E \operatorname{Tr}\left[\left(i \delta_{\alpha \delta} \Gamma_{\alpha} G^{r}-i \delta_{\alpha \gamma} \Gamma_{\delta} G^{a}\right.\right. \\
& \left.-\Gamma_{\delta} G^{a} \Gamma_{\alpha} G^{r}\right)\left(i \delta_{\beta \gamma} \Gamma_{\beta} G^{r}-i \delta_{\beta \delta} \Gamma_{\gamma} G^{a}\right. \\
& \left.\left.-\Gamma_{\gamma} G^{a} \Gamma_{\beta} G^{r}\right)\right] f_{\gamma}\left(1-f_{\delta}\right),
\end{aligned}
$$

where $G^{a}=G^{a}(E, U)$ is the advanced Green's function, $f_{\gamma}$ $\equiv f\left(E-q V_{\gamma}\right)$ is the Fermi distribution function with $V_{\gamma}$ the bias potential at probe $\gamma$, and $\Gamma_{\alpha} \equiv \Gamma_{\alpha}\left(E-q V_{\alpha}\right)$ is the linewidth function.

A crucial next step is to calculate the potential landscape $U(\mathbf{r})$, which appears explicitly in the Green's functions. $U(\mathbf{r})$ is determined self-consistently by Poisson equation

$$
\nabla^{2} U=4 \pi i q \int(d E / 2 \pi) G^{<}(E, U),
$$

where the boundary condition is such that $U \rightarrow V_{\alpha}$ at probe $\alpha$, and the lesser Green's function $G^{<}$is related to the retarded and advanced Green's functions $G^{r}$ and $G^{a}$,

$$
G^{<}(E, U)=G^{r} \sum_{\beta} i \Gamma_{\beta}\left(E-q V_{\beta}\right) f\left(E-q V_{\beta}\right) G^{a} .
$$

Equation (7) is a nonlinear equation because $G^{r, a}$ depends on $U(\mathbf{r})$ [see Eq. (2)]. By self-consistently solving Eqs. (2), (7), and (8), we obtain the Green's functions as well as the internal potential $U$. We can then calculate shot noise from Eq. (6), which is now a nonlinear function of the external bias $\left\{V_{\alpha}\right\}$. This theoretical procedure can be carried out at least numerically, but in this work we are interested in cases where analytical derivations are possible.

For a two-probe system, Eq. (6) reduces to

$$
\begin{aligned}
\left\langle(\Delta I)^{2}\right\rangle= & \frac{q^{2}}{\pi} \Delta \nu \int d E\left\{\left[f_{1}\left(1-f_{1}\right)+f_{2}\left(1-f_{2}\right)\right] \operatorname{Tr}[\hat{T}]\right. \\
& \left.+\left(f_{1}-f_{2}\right)^{2} \operatorname{Tr}[(1-\hat{T}) \hat{T}]\right\},
\end{aligned}
$$

where $\hat{T}(E, U)=\Gamma_{1} G^{r} \Gamma_{2} G^{a}$ is the transmission operator such that $\operatorname{Tr}[\hat{T}(E, U)]$ is the transmission coefficient; here the trace is over the matrices written in real space.

To end this section, we discuss the gauge-invariance condition. It is easy to prove that the noise spectra [Eqs. (6) and (9)] are gauge invariant: shifting the potential everywhere by a constant $V_{o}, U \rightarrow U+V_{o}$ and $V_{\alpha} \rightarrow V_{\alpha}+V_{o} ;\left\langle(\Delta I)^{2}\right\rangle$ calculated from these expressions remains the same. It is useful to note that in Eqs. (6) and (9) the quantity $\Gamma$ depends on bias voltage: without such a voltage dependence the gauge invariance cannot be satisfied.

\section{THE WIDEBAND LIMIT}

In the commonly used wideband limit, ${ }^{28}$ the coupling matrix $\Gamma$ is assumed to be independent of energy. This limit corresponds to cases where the probes have no feature; thus the internal potential $U(\mathbf{r})$ becomes a space-independent constant $U_{0}$ (the value of $U_{0}$ depends on bias voltages $\left\{V_{\alpha}\right\}$ and still needs to be determined). For a single level system, as far as the nonlinear current-voltage curve is concerned, wideband limit corresponds to a resonance tunneling system where the scattering matrix takes a Breit-Wigner form.

In wideband limit the steady state Green's function is given by $G_{0}^{r}=1 /\left(E-E_{0}+i \Gamma / 2\right)$; for this Green's function the integral in Eq. (9) can be done exactly at zero temperature. We obtain 


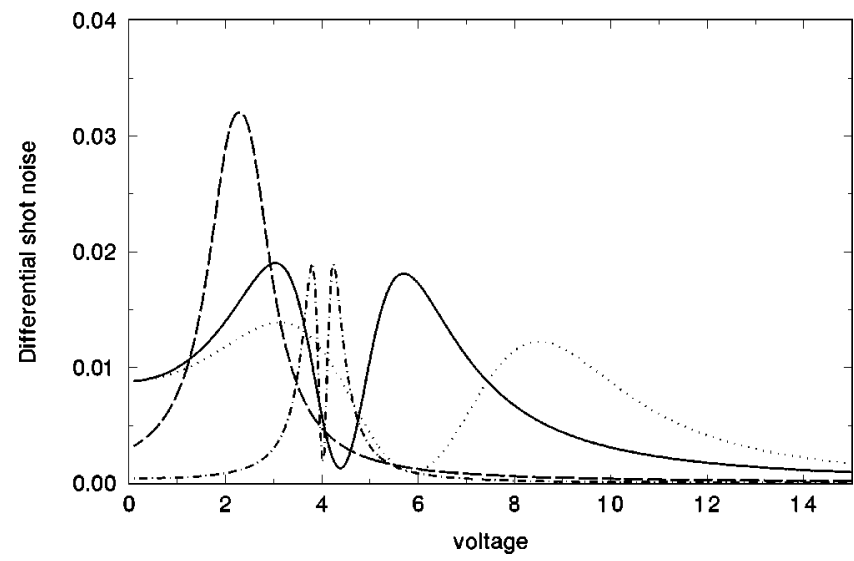

FIG. 1. The differential shot noise versus the voltage. Solid line, $\Gamma_{1}=\Gamma_{2}=0.5$ and $C_{1}=C_{2}=0.5$; dotted line, $\Gamma_{1}=\Gamma_{2}=0.5$ and $C_{1}$ $=C_{2}=0.1$; dot-dashed line, $\Gamma_{1}=\Gamma_{2}=0.1$ and $C_{1}=C_{2}=0.4$; and dashed line, $\Gamma_{1}=0.1, \Gamma_{2}=0.8, C_{1}=0.1$, and $C_{2}=0.8$. Here $E_{F}$ $-E_{0}=-2.0$.

$$
\begin{aligned}
\left\langle(\Delta I)^{2}\right\rangle= & \frac{q^{2}}{\pi} \Delta \nu \frac{4 \Gamma_{1}^{2} \Gamma_{2}^{2}}{\Gamma^{3}}\left\{\frac { \Gamma _ { 1 } ^ { 2 } + \Gamma _ { 2 } ^ { 2 } } { 2 \Gamma _ { 1 } \Gamma _ { 2 } } \left[\arctan \left(\frac{\Delta E_{1}}{\Gamma / 2}\right)\right.\right. \\
& \left.-\arctan \left(\frac{\Delta E_{2}}{\Gamma / 2}\right)\right]-\frac{\Gamma}{2}\left[\frac{\Delta E_{1}}{(\Gamma / 2)^{2}+\left(\Delta E_{1}\right)^{2}}\right. \\
& \left.\left.-\frac{\Delta E_{2}}{(\Gamma / 2)^{2}+\left(\Delta E_{2}\right)^{2}}\right]\right\},
\end{aligned}
$$

where $\Delta E_{\beta}=E_{F}-E_{0}-q U_{0}+q V_{\beta}$.

While the internal potential $U_{0}$ can be determined by the Poisson equation (7), which requires numerical analysis, we instead parametrize it by geometrical capacitances $C_{1}$ and $C_{2}$ of the left and the right coupling regions (regions where the conductor connects to the two probes). The charge in the well due to the Coulomb interaction is given by ${ }^{29}$

$$
\begin{aligned}
\Delta Q & =-i \int(d E / 2 \pi)\left[G^{<}\left(E, U_{0}\right)-G_{0}^{<}\right] \\
& =C_{1}\left(U_{0}-V_{1}\right)+C_{2}\left(U_{0}-V_{2}\right),
\end{aligned}
$$

where $\Delta Q$ is the total charge in the well and $G_{0}^{<}$is the equilibrium lesser Green's function. In the wideband limit, this equation reduces to

$$
\begin{array}{r}
\sum_{\beta} \Gamma_{\beta} \arctan \left(\frac{\Delta E_{\beta}}{\Gamma / 2}\right)-\Gamma \arctan \left(\frac{E_{F}-E_{0}}{\Gamma / 2}\right) \\
=\frac{\pi \Gamma}{q}\left[C_{1}\left(U_{0}-V_{1}\right)+C_{2}\left(U_{0}-V_{2}\right)\right] .
\end{array}
$$

When $C_{1}=C_{2}=0$, Eq. (12) corresponds to the quasineutrality approximation that neglects the charge polarization in the system. Hence, using two phenomenological constants $C_{1}$ and $C_{2}$, we determine $U_{0}$ from the last equation. The noise spectra of Eq. (10) is now completely specified.

Figure 1 plots the nonlinear shot noise in terms of its differential $d\left\langle(\Delta I)^{2}\right\rangle / d V$ as a function of bias for four different sets of parameters: symmetric structures with $\Gamma_{1}$ $=\Gamma_{2}=0.5, \quad C_{1}=C_{2}=0.5 \quad($ solid line $) ; \Gamma_{1}=\Gamma_{2}=0.5, \quad C_{1}$

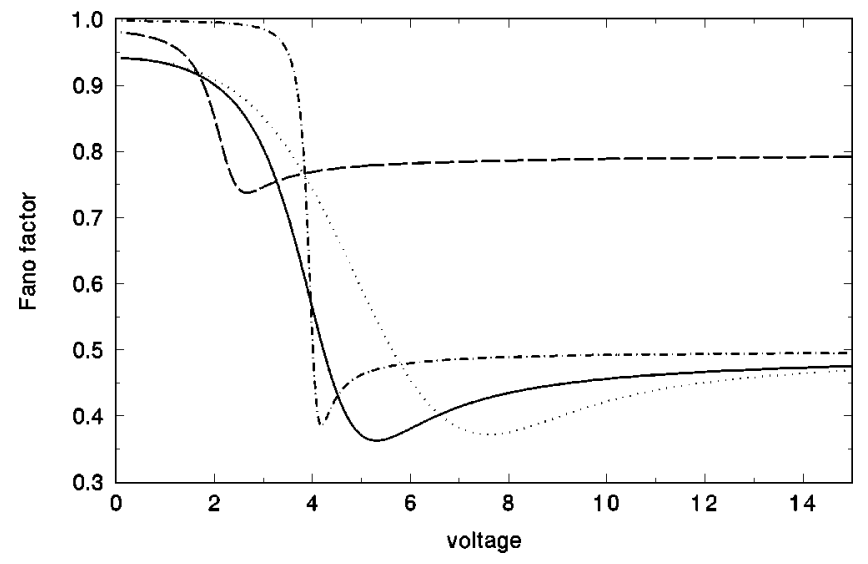

FIG. 2. The corresponding Fano factor of Fig. 1.

$=C_{2}=0.1$ (dotted line); $\Gamma_{1}=\Gamma_{2}=0.1, C_{1}=C_{2}=0.4$ (dotdashed line); and an asymmetric structure with $\Gamma_{1}=0.1, \Gamma_{2}$ $=0.8, C_{1}=0.1$, and $C_{2}=0.8$ (dashed line). For symmetric structures, we always observe two peaks for the differential nonlinear noise whereas for the asymmetric structure there is only one. This can be understood qualitatively as follows. Since the shot noise at small voltage is proportional to ${ }^{9} T$ $-T^{2}$, the suppression reaches maximum near the resonant point for the symmetric structure because $T \approx T^{2} \approx 1$ at resonance. As a result, a peak appears on each side of the resonant point giving rise to the two peaks in Fig. 1. For an asymmetric structure the shot-noise suppression is not as strong as that of the symmetric case (see Fig. 2). For a strongly asymmetric structure such as that studied here, the quantum resonance is weak resulting to only one peak in the differential noise spectra. For the symmetric system, the separation between the two peaks is proportional to $\Gamma$ (Fig. $1)$. An effect of Coulomb interaction is to shift the resonance energy $E_{0}$ to higher values $E_{0}+q U_{0}$. For the solid and dotted curves in Fig. 1, these resonances occur near voltages 4 and 6 , respectively (the dip position). Smaller capacitance coefficients (dotted line) correspond to larger internal potential $U_{0}$; hence the resonance position for the dotted curve (with smaller capacitance coefficients) is shifted further relative to the solid curve (with larger capacitances).

As discussed in the Introduction, the classical shot noise is given by $2 q I \Delta \nu$. The deviation from this classical value is characterized by the Fano factor, which is defined as

$$
\gamma \equiv \frac{\left\langle(\Delta I)^{2}\right\rangle}{2 q I \Delta \nu},
$$

where the current $I$ in wideband limit can be derived as

$$
I=\frac{q \Gamma_{1} \Gamma_{2}}{\pi \Gamma}\left[\arctan \left(\frac{2 \Delta E_{1}}{\Gamma}\right)-\arctan \left(\frac{2 \Delta E_{2}}{\Gamma}\right)\right] .
$$

Before presenting the plot of $\gamma$ for our nonlinear analysis, two observations are in order. First, it is easy to show that in the limit $\left(V_{1}-V_{2}\right) \rightarrow 0$ and $\Gamma \rightarrow 0, \gamma \rightarrow 1$. Second, in the opposite limit of large bias, the Fano factor is reduced to the known expression ${ }^{21} \gamma=\left(\Gamma_{1}^{2}+\Gamma_{2}^{2}\right) / \Gamma^{2}$. For symmetrical systems $\left(\Gamma_{1}=\Gamma_{2}\right)$, the large bias limit gives $\gamma=0.5$. The same behavior has been observed in the tunneling experiment ${ }^{15}$ away from the NDR region. Physically, currents coming 


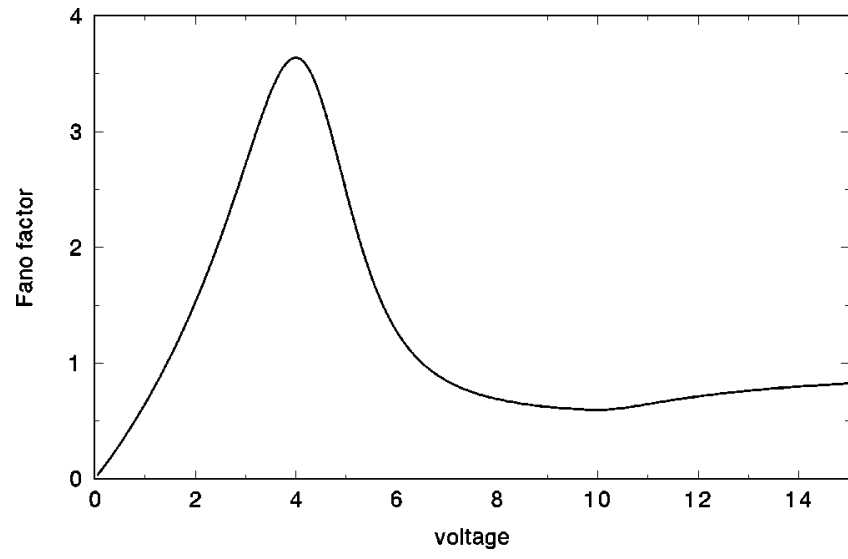

FIG. 3. The Fano factor versus the voltage when the energy cutoff is introduced for $\Gamma_{1}=\Gamma_{2}=0.5, C_{1}=C_{2}=0.5$, and $E_{F}-E_{0}$ $=-2.0$.

from both leads contribute to the Fano factor, but at large bias the current coming from the low-voltage lead can be neglected, thus shot noise is suppressed.

Figure 2 plots the Fano factor versus voltage for the same system parameters as those of Fig. 1. As expected, the Fano factor approaches 0.5 for symmetric structures at large voltage. We also observe that the Fano factor takes minimum value near resonance: in the wideband limit $\gamma$ can be smaller than 0.5. For smaller $\Gamma$, the transition from $\gamma \approx 1$ to $\gamma \approx 0.5$ is much sharper (dot-dashed line). This result suggests a more pronounced noise reduction for conductors that are more weakly coupled to the leads (smaller $\Gamma$ ). On the other hand, for the asymmetric case the suppression of Fano factor is not as strong (dashed line) due to weaker quantum resonance.

The wideband limit of the theory does not allow negative differential resistance, ${ }^{28}$ hence Fig. 2 does not show enhancement of shot noise that has been observed in the experiment of Ref. 15. To obtain NDR within the wideband approximation, we assume the leads to have a finite occupied bandwidth by introducing an energy cutoff in the integration of Eq. (10), as suggested by Jauho, Wingreen, and Meir. ${ }^{28}$ This simple procedure indeed produced a Fano factor that can be greater than unity, as shown in Fig. 3. Our analysis thus reconfirms that shot noise can be enhanced by the existence of a NDR region. The experimental result ${ }^{15}$ showed a sharper increase of Fano factor when bias is varied from that of Fig. 3. This discrepancy suggests a need to go beyond wideband limit in order to obtain a quantitative agreement.

\section{WEAKLY NONLINEAR LIMIT}

The wideband limit discussed above reduces the system to essentially a single level inside a zero-dimensional quantum dot, this allows us to obtain closed-form results for the full nonlinear shot-noise spectra including large bias voltages. In this section we examine another limit, namely the weakly nonlinear limit where the bias is finite but not large. In this case we can expand the shot-noise formula order by order in bias and derive the weakly nonlinear shot-noise spectral coefficients. In particular, we expand the noise spectra [Eq. (9)] in the form

$$
\begin{aligned}
\left\langle(\Delta I)^{2}\right\rangle= & P_{0}+P_{1}\left(V_{1}-V_{2}\right)+P_{2}\left(V_{1}-V_{2}\right)^{2} \\
& +P_{3}\left(V_{1}-V_{2}\right)^{3}+\cdots,
\end{aligned}
$$

where the equilibrium noise $P_{0}$ and the linear noise spectra $P_{1}$ have been considered in detail before. ${ }^{9}$ Here we derive an expression for the second-order nonlinear noise spectra $P_{2}$ at zero temperature.

To proceed, we first determine the internal potential $U$. Instead of applying the phenomenological charging model to parametrize $U$ in terms of capacitance coefficients, in weakly nonlinear analysis, we solve $U$ self-consistently order by order in bias in exactly the same way as documented previously for nonlinear analysis of $I$ - $V$ curves. ${ }^{30,31}$ Summarizing briefly, we expand $U$ in powers of voltages,

$$
U=U_{\mathrm{eq}}+\sum_{\alpha} u_{\alpha} V_{\alpha}+\frac{1}{2} \sum_{\alpha \beta} u_{\alpha \beta} V_{\alpha} V_{\beta}+\cdots
$$

where $U_{\text {eq }}$ is the equilibrium potential and $u_{\alpha}(\mathbf{r}), u_{\alpha \beta} \ldots(\mathbf{r})$ are the characteristic potentials. ${ }^{30,31}$ They are determined by Poisson-like equations that are derived by expanding Eq. (7) in powers of voltage. ${ }^{30,31}$ These procedures have been discussed in Ref. 30 for linear characteristic potential $u_{\alpha}$ and in Ref. 31 for nonlinear characteristic potentials $u_{\alpha \beta} \ldots$. We refer interested readers to these references for details, and from now on we assume that the characteristic potentials have been obtained.

At zero temperature the shot-noise formula [Eq. (9)] reduces to

$$
\left\langle(\Delta I)^{2}\right\rangle=\Delta \nu \frac{q^{2}}{\pi} \int_{E_{F}+q V_{2}}^{E_{F}+q V_{1}} d E \operatorname{Tr}[(1-\hat{T}) \hat{T}] .
$$

Denoting $g(E, U) \equiv(1-\hat{T}) \hat{T}$, we expand $g(E, U)$ with respect to $E$ and $V$,

$$
\begin{aligned}
g(E, U) & \approx g(E, 0)+\frac{d g(E, 0)}{d U} U \\
& \approx g_{0}+\frac{d g_{0}}{d E}\left(E-E_{F}\right)+\frac{d g_{0}}{d U} U,
\end{aligned}
$$

where $U$ is the diagonal matrix for the internal potential, $g_{0}=g\left(E_{F}, 0\right)$ and $\left(d g_{0} / d U\right) U \equiv \Sigma_{x}\left[\delta g_{0} / \delta U(x)\right] U(x)$. Substituting Eq. (17) into Eq. (16) and completing the energy integration, we obtain the second-order noise term

$$
\begin{aligned}
P_{2}\left(V_{1}-V_{2}\right)^{2}= & \Delta \nu \frac{q^{2}}{\pi} \operatorname{Tr}\left[\frac{q^{2}}{2} \frac{d g_{0}}{d E}\left(V_{1}^{2}-V_{2}^{2}\right)\right. \\
& \left.+\frac{d g_{0}}{d U} q\left(u_{1} V_{1}+u_{2} V_{2}\right)\left(V_{1}-V_{2}\right)\right] .
\end{aligned}
$$

Using known relations ${ }^{23} \quad q d g_{0} / d E=-d g_{0} / d U$ and $\Sigma_{\alpha} u_{\alpha}(\mathbf{r})=1$, we derive

$$
P_{2}=\Delta \nu \frac{q^{3}}{2 \pi} \operatorname{Tr}\left[\frac{d g_{0}}{d U}\left(2 u_{1}-1\right)\right] .
$$


Following similar procedure one can derive higher-order nonlinear shot-noise coefficients. For instance, the thirdorder nonlinear noise spectra is found to be

$$
P_{3}=\Delta \nu \frac{q^{3}}{6 \pi} \operatorname{Tr}\left[3 q \frac{d g_{0}}{d U} u_{11}+\frac{d^{2} g_{0}}{d U^{2}}\left(1-3 u_{1}+3 u_{1}^{2}\right)\right]
$$

As an explicit example of the general expressions (19) and (20), let us derive $P_{2}$ and $P_{3}$ for a resonance tunneling system near a resonance point $E_{0}$. In this situation the function $g(E, U)$ of Eq. (17) can be calculated from the BreitWigner form ${ }^{23}$ of the scattering matrix, $s_{\alpha \beta}(E) \sim\left(\delta_{\alpha \beta}\right.$ $-i \sqrt{\Gamma_{\alpha} \Gamma_{\beta}} / \Delta$ ), where $\Gamma_{\alpha}$ is the decay width of barrier $\alpha$, $\Delta=E-E_{0}+i \Gamma / 2$ with $\Gamma=\Gamma_{1}+\Gamma_{2}$. Next, the characteristic potentials can be derived in quasineutrality approximation, ${ }^{23,31}$ which gave $u_{1}=\Gamma_{1} / \Gamma$ and $u_{11}=-2(E$ $\left.-E_{0}\right) T / \Gamma^{2}$. We thus obtain

$$
P_{2}=\Delta \nu \frac{q^{4}}{2 \pi} \frac{\Gamma_{2}-\Gamma_{1}}{\Gamma}(1-2 T) \frac{d T}{d E}
$$

and

$$
\begin{aligned}
P_{3}= & -\Delta \nu \frac{q^{5}}{6 \pi}\left\{6 \frac{E-E_{0}}{\Gamma^{2}} T(2 T-1) \frac{d T}{d E}\right. \\
& \left.+\frac{\Gamma_{1}^{2}+\Gamma_{2}^{2}-\Gamma_{1} \Gamma_{2}}{\Gamma^{2}}\left[(2 T-1) \frac{d^{2} T}{d E^{2}}+2\left(\frac{d T}{d E}\right)^{2}\right]\right\},
\end{aligned}
$$

where $T=\Gamma_{1} \Gamma_{2} /|\Delta|^{2}$. Clearly, $P_{2}=0$ while $P_{3} \neq 0$ for a symmetric system for which $\Gamma_{1}=\Gamma_{2}$. In general, all the coefficients of even power of bias vanish for symmetric systems since shot noise cannot change when bias changes sign. It is known that resonance tunneling with Breit-Wigner scattering matrix is equivalent to the wideband limit in NEGF analysis. One can straightforwardly confirm that expressions (21) and (22) are indeed obtained by expanding NEGF results (10) and (12) to the appropriate order in bias. This gives strong confirmation on the validity of weakly nonlinear analysis presented in this section.

\section{SUMMARY}

In this work we have developed a general nonlinear $\mathrm{dc}$ theory for calculating the shot-noise spectra in the mesoscopic regime. The framework is based on nonequilibrium Green's functions with the important extension of solving the internal potential self-consistently. A direct advantage of our method is that the final expression for shot noise becomes gauge invariant, which is an essential requirement for a nonlinear transport theory. Equations (6), (2), and (7) completely determine the nonlinear shot-noise spectra of an arbitrary multiprobe conductor; they form the basic results of our theory. Practically, one must solve the quantum scattering problem, which gives the Green's functions, in conjunction with the Poisson equation. Technically, these expressions form a convenient basis for numerical predictions of shot-noise spectra at finite bias voltages. For instance, one can easily compute various Green's functions and the coupling matrix $\Gamma$ for multiprobe conductors using tight-binding models $;{ }^{25}$ and the Poisson equation can be solved in real space using very powerful numerical techniques. ${ }^{32}$

In the wideband limit and the weakly nonlinear limit, the basic equations (6), (2), and (7) can be analyzed in closed form. The nonlinear theory reveals that the shot noise of a mesoscopic conductor can be quite sensitive to the external bias strength, and in general the suppression of noise is most efficient near a quantum resonance point and is stronger for symmetric systems than asymmetric systems. The suppression is also more efficient for conductors weakly coupled to the leads. In the negative differential resistance region of the nonlinear current-voltage characteristics, our result confirms the existence of shot-noise enhancement, which has been observed experimentally. For weakly nonlinear transport regime, we have derived the shot-noise nonlinear coefficients order by order in bias, and these coefficients should be adequate when the external bias is finite but not large.

\section{ACKNOWLEDGMENTS}

We thank Professor T.H. Lin and Q.F. Sun for helpful discussions concerning the derivation of Eq. (6) using NEGF as well as a number of other issues of the formalism. We gratefully acknowledge support by a CRCG grant from the University of Hong Kong. H.G. was supported by NSERC of Canada and FCAR of Québec. We thank the computer center of HKU for computational facilities.
${ }^{1}$ M. J. M. de Jong and C. W. J. Beenakker, in Mesoscopic Electron Transport, Vol. 345 of NATO Advanced Studies Institute, Series E, edited by L.L. Sohn, L.P. Kouwenhoven, and G. Schön (Kluwer, Dordrecht, 1997), p. 225.

${ }^{2}$ M. Büttiker, J. Math. Phys. 37, 4793 (1996).

${ }^{3}$ M. Henny, S. Oberholzer, C. Struck, T. Heinzel, K. Ensslin, M. Holland, and C. Schönenberger, Science 284, 296 (1999); W. D. Oliver, J. Kim, Robert C. Liu, and Y. Yamamoto, ibid. 284, 299 (1999).

${ }^{4}$ M. Henny, S. Oberholzer, C. Struck, and C. Schönenberger, Phys. Rev. B 59, 2871 (1999).

${ }^{5}$ R. Landauer, Nature (London) 392, 658 (1998).
${ }^{6}$ T. Gramespacher and M. Büttiker, Phys. Rev. Lett. 81, 2763 (1998).

${ }^{7}$ J. R. Pierce, Bell Syst. Tech. J. 27, 158 (1948).

${ }^{8}$ V. A. Khlus, Zh. Eksp. Teor. Fiz. 93, 2179 (1987) [Sov. Phys. JETP 66, 1243 (1987)].

${ }^{9}$ M. Büttiker, Phys. Rev. Lett. 65, 2901 (1990); Phys. Rev. B 46, 12485 (1992).

${ }^{10}$ Y. P. Li, D. C. Tsui, J. J. Heremans, J. A. Simmons, and G. W. Weimann, Appl. Phys. Lett. 57, 774 (1990).

${ }^{11}$ M. Reznikov, M. Heiblum, H. Shtrikman, and D. Mahalu, Phys. Rev. Lett. 75, 3340 (1995).

${ }^{12}$ A. Kumar, L. Saminadayar, D. C. Glattli, Y. Jin, and B. Etienne, 
Phys. Rev. Lett. 76, 2778 (1996).

${ }^{13}$ T. Gonzalez, C. González, J. Mateos, D. Pardo, L. Reggiani, O. M. Bulashenko, and J. M. Rubi, Phys. Rev. Lett. 80, 2901 (1998).

${ }^{14}$ C. W. Beenakker, Phys. Rev. Lett. 82, 2761 (1999).

${ }^{15}$ G. Iannaccone, G. Lombardi, A. Macucci, and B. Pellegrini, Phys. Rev. Lett. 80, 1054 (1998).

${ }^{16}$ V. V. Kuznetsov, E. E. Mendez, J. D. Bruno, and J. T. Pham, Phys. Rev. B 58, R10 159 (1998).

${ }^{17}$ G. Iannaccone, M. Macucci, and B. Pellegrini, Phys. Rev. B 55, 4539 (1997).

${ }^{18}$ Y. M. Blanter and M. Büttiker, Phys. Rev. B 59, 10217 (1999).

${ }^{19}$ K. E. Nagaev, Phys. Lett. A 169, 103 (1992); Phys. Rev. B 57, 4628 (1998).

${ }^{20}$ M. J. M. de Jong and C. W. Beenakker, Phys. Rev. B 51, 16867 (1995).

${ }^{21}$ L. Y. Chen and C. S. Ting, Phys. Rev. B 43, 4534 (1991).

${ }^{22}$ G. H. Ding and T. K. Ng, Phys. Rev. B 56, R15 521 (1997).
${ }^{23}$ For a review, see M. Büttiker and T. Christen, in Quantum Transport in Semiconductor Submicron Structures, edited by B. Kramer (Kluwer Academic, Dordrecht, 1996), p. 263.

${ }^{24}$ T. Gramespacher and M. Büttiker, Phys. Rev. B 56, 13026 (1997).

${ }^{25}$ S. Datta, Electronic Transport in Mesoscopic Systems (Cambridge University Press, New York, 1995).

${ }^{26}$ D. S. Fisher and P. A. Lee, Phys. Rev. B 23, 6851 (1981).

${ }^{27}$ It is also possible to directly derive Eq. (6) from NEGF.

${ }^{28}$ A. P. Jauho, N. S. Wingreen, and Y. Meir, Phys. Rev. B 50, 5528 (1994).

${ }^{29}$ M. H. Pedersen and M. Büttiker, Phys. Rev. B 58, 12993 (1998).

${ }^{30}$ M. Büttiker, J. Phys.: Condens. Matter 5, 9361 (1993).

${ }^{31}$ Z. S. Ma, J. Wang, and H. Guo, Phys. Rev. B 57, 9108 (1998); 59, 7575 (1999).

${ }^{32}$ J. Wang, H. Guo, J. L. Mozos, C. C. Wan, G. Taraschi, and Q. R. Zheng, Phys. Rev. Lett. 80, 4277 (1998). 